\title{
Ejakulation unter Kontrolle
}

- Wenn eine Ejaculatio praecox, auch „premature ejaculation“ (PE) genannt, diagnostiziert wurde, sollte in Absprache mit dem Patienten rasch eine Therapie aufgenommen werden, um negative psychische und partnerschaftliche Konsequenzen zu vermeiden. Seit kurzer Zeit steht mit Dapoxetin (Priligy ${ }^{\circledR}$ ) das erste und einzige zugelassene orale Medikament zur Behandlung des vorzeitigen Samenergusses zur Verfügung. Dapoxetin ist ein kurzwirksamer Serotonin-Wiederaufnahmehemmer, der speziell für die Therapie der PE entwickelt wurde. Eine pharmakologische Besonderheit, die Dapoxetin auszeichnet, ist das schnelle An- und Abfluten: Bereits circa eine Stunde nach Einnahme einer Einzeldosis von $30 \mathrm{mg}$ oder $60 \mathrm{mg}$ werden maximale Plasmakonzentrationen erreicht. Innerhalb von 24 Stunden fallen diese wieder auf unter $5 \%$ des Maximalwertes zurück. Aufgrund dieser Eigenschaft ist es möglich, Dapoxetin ungefähr ein bis drei Stunden vor dem Geschlechtsverkehr einzunehmen.

Die Wirksamkeit von Dapoxetin wurde in klinischen Studien mit mehr als 6.000 Patienten geprüft. Dabei zeigte es sich, dass dieser Serotonin-Wiederaufnahmehemmer die Symptome der PE im Vergleich zu Placebo signifikant verbessern konnte. Durch die Einnahme des Wirkstoffs verlängerte sich die Zeit bis zum Samenerguss um mehr als das Dreifache. Nach 24 Wochen wurden die Teilnehmer zudem nach ihrer Zufriedenheit mit dem Geschlechtsverkehr befragt: 48,5\% (30 mg Dapoxetin) und 55,8\% (60 mg Dapoxetin) der Verum-Gruppe antworteten, sie seien zufriedener mit ihrem

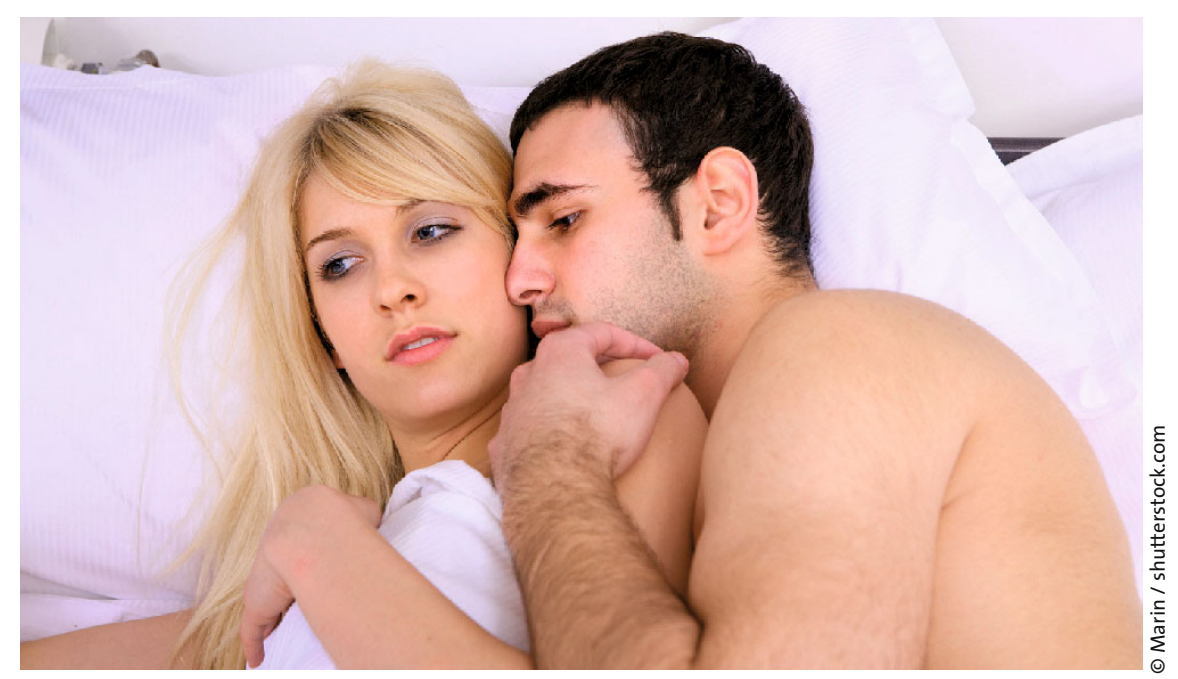

Eine Ejaculatio praecox stellt eine Partnerschaft häufig auf die Probe.
Geschlechtsverkehr als zu Studienbeginn. In der Placebo-Gruppe machten nur 35,7\% der Teilnehmer diese Angabe.

Die Therapie mit Dapoxetin kann also dazu beitragen, die sexuelle Gesamtzufriedenheit der PE-Patienten signifikant zu verbessern.

Nach Informationen von Janssen-Cilag, Neuss

\section{Inkontinenztherapie: Wirksamkeit und Verträglichkeit entscheidend}

— Wirksamkeit, Verträglichkeit und eine hohe Compliance sind für Urologen die wichtigsten Eigenschaften zur Therapie der Dranginkontinenz. Dies zeigt eine Befragung von 100 Urologen im Auftrag des Arzneimittelherstellers Madaus im September 2009. Genau diesen Anforderungen entspricht das Eigenschaftsprofil der Trospiumchlorid 60 mg Retardkapseln. Ein Großteil der befragten Ärzte sieht bei Urive$\mathrm{sc}^{\circledR}$ Vorteile im Vergleich zu anderen Spasmolytika, hierbei vor allem durch die einmal tägliche Einnahme.

Befragt nach der Relevanz verschiedener Eigenschaften von Präparaten zur Therapie der Dranginkontinenz, nennen die Urologen als die wichtigsten: eine gute Wirksamkeit (96\% der Verordner bzw. 100\% der Nochnicht-Verordner), eine gute Verträglichkeit (95\% bzw. 93\%) sowie eine hohe Compliance der Patienten (93\% bzw. 89\%). Das
Eigenschaftsprofil von Urivesc ${ }^{\circledR}$ erfüllt diese Ansprüche: Die neue Galenik der 60 mg Retardkapseln ermöglicht nicht nur die patientenfreundliche tägliche Einmalgabe, sondern sie bedingt auch das günstigere Verhältnis von Wirksamkeit und Nebenwirkungsprofil. Dieses ist gegenüber anderen Anticholinergika signifikant verbessert: So ist Mundtrockenheit, die häufigste Nebenwirkung bei der Behandlung der Harninkontinenz, bei Urivesc ${ }^{\circledR}$ mit 10,7\% im Vergleich zu anderen Präparaten mit am niedrigsten. Die Häufigkeit von Obstipation als ein weiteres unerwünschtes Ereignis ist ebenfalls niedriger.Zudem werden durch die günstige Pharmakokinetik von Trospiumchlorid - der Wirkstoff ist hydrophil und daher kaum ZNS-gängig - kognitive Einschränkungen wie Tagesschläfrigkeit reduziert.

Umfangreiche Phase-III-Studien bei Patienten mit reiner Dranginkontinenz und
Mischinkontinenz belegen außerdem die gute Wirksamkeit von Urivesc ${ }^{\circledR}$ : In zwölf Wochen reduzierten sich in der Verumgruppe die Miktionshäufigkeit pro Tag um 20,5\% (gegenüber $14 \%$ in der Kontrollgruppe) sowie die täglichen Dranginkontinenzepisoden um bis zu $80 \%$ (vs. 55,6\%).

Die nur einmal tägliche Einnahme sowie die verringerten Nebenwirkungen von Urives ${ }^{\circledR}$ erhöhen zudem die Therapietreue der Patienten deutlich.

Mit Tagestherapiekosten von 1 EUR ist Urivesc $^{\circledR}$ das günstigste Präparat unter den oralen Einmalgaben zur Therapie der überaktiven Blase. Es ist mindestens 35\% günstiger als andere Einmalgaben und somit deutlich budgetschonend.

Nach Informationen von

Rottapharm|Madaus, Köln 